\title{
DOE/ER/40293--9
}

Middle Tennessee State University

DE-FG05-86ER40293-9

\section{NUCLEAR STRUCTURE STUDIES via NEUTRON INTERACTIONS}

Continuation Progress Report

1 July 1994 - 30 June 1995
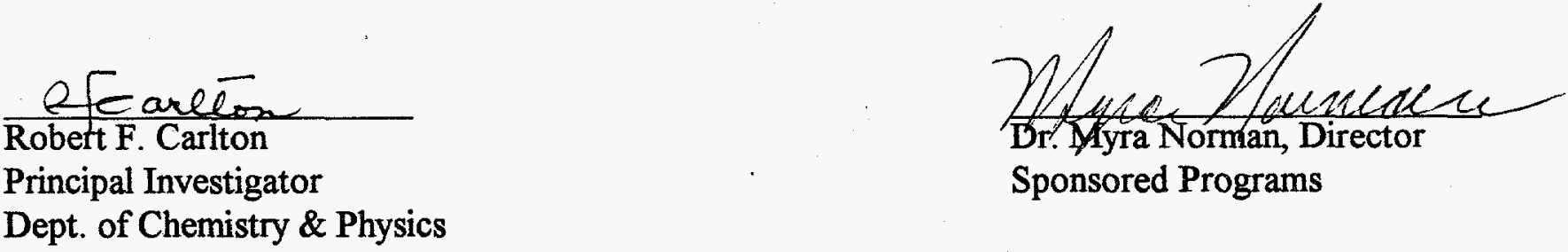

Sponsored Programs

Dept. of Chemistry \& Physics

Middle Tennessee State University

Murfreesboro, Tennessee 37132

January 1995

Prepared for

THE U.S. DEPARTMENT OF ENERGY

Under Contract No. DE-FG05-86ER40293 


\section{DISCLAIMER}

This report was prepared as an account of work sponsored by an agency of the United States Government. Neither the United States Government nor any agency thereof, nor any of their employees, make any warranty, express or implied, or assumes any legal liability or responsibility for the accuracy, completeness, or usefulness of any information, apparatus, product, or process disclosed, or represents that its use would not infringe privately owned rights. Reference herein to any specific commercial product, process, or service by trade name, trademark, manufacturer, or otherwise does not necessarily constitute or imply its endorsement, recommendation, or favoring by the United States Government or any agency thereof. The views and opinions of authors expressed herein do not necessarily state or reflect those of the United States Government or any agency thereof. 


\section{DISCLAIMER}

Portions of this document may be illegible in electronic image products. Images are produced from the best available original document. 


\section{ABSTRACT}

Neutron total cross section measurements of ${ }^{122} \mathrm{Sn}$ has been analyzed over the energy range 0.013 to $0.310 \mathrm{MeV}$. The $R-$ matrix analysis has resulted in resonance and average parameters which provide for a complete representation of the neutron entrance channels for the $s_{1 / 2}, p_{1 / 2}$, and $p_{3 / 2}$ contributions. The $s$ - and $p$ - neutron strength functions have been determined to be $0.123 \pm 0.023$ and $2.0 \pm 0.200$, respectively (in units of $10^{-4}$ ). Limits are placed on level spacings and strength functions for the individual partial wave components. The $s$ - wave potential scattering radius has been determined to be $6.3 \pm 0.1 \mathrm{fm}$. Average scattering functions, deduced from the average parameters, have been used to determine the real well depth of an optical model potential which reproduces these functions. We find, as have others, that the real well depth is parity dependent.

Two computer codes were developed: (1) a minimization program for finding parameters of a dispersive optical model which reproduce the high resolution data of the ORELA facility, and (2) an X-windows graphical user interface has been developed to permit interactive resonance analysis of the neutron total cross section data using analysis-and-minimization codes developed previously. These will contribute to the more routine analysis of ORELA data.

\section{Introduction}

The isotopes of tin have been the subject of numerous investigations because of the possibility of systematic studies of nuclear structure and the magic and near-magic number of nucleons and the consequent simplification of model investigations. Fuketa et al. ${ }^{1}$ made the first systematic measurements of neutron transmission measurements of all of the tin isotopes many years ago at the Oak Ridge chopper up to a neutron energy of $10 \mathrm{keV}$. Though these measurements revealed few resonances above $2 \mathrm{keV}$, many interesting features were observed in this narrow energy interval. They found the s-wave strength functions to be lower than both that for other elements in this mass region, and predictions based on an optical model with high absorption at the nuclear surface. Harvey et. al. ${ }^{2}$ confirmed strong isolated p-wave resonances and associated $M I$ radiation in several of the isotopes. Nuclear levels in the unbound region are not well explored for tin. In the present work we have used high resolution neutron total cross section measurements at energies above $10 \mathrm{keV}$, where one expects other than s-wave interaction, to identify the positions and strengths of $1 / 2^{+}$, $1 / 2^{-}$, and $3 / 2^{-}$levels in this region. This extension to higher energies will provide information for the refinement of model calculations and permit the investigation of possible doorway structure in the s-wave interaction channel.

Another useful feature of the $\mathrm{Sn}$ isotopes is the provision offered for studying the isospin dependence of various nuclear properties. Numerous scattering and total cross section measurements at both low and high neutron energies have been carried out and optical model parameters deduced from fits to the data. None of these studies have resolved $\ell$ - and $\mathrm{J}$ specific contributions. Only in the process of fitting the total data set have $s-$ and $p$ - wave strength functions and scattering radii been deduced in some cases.

Most recently a study ${ }^{3}$ of ${ }^{116,120} \mathrm{~S} n$ differential elastic and inelastic scattering cross sections and analyzing powers in the energy range, $10-24 \mathrm{MeV}$ have been analyzed together with total cross section measurements from $10 \mathrm{keV}$ to 100
$\mathrm{MeV}$ to more definitively determine the parameters of a deformed optical model potential to be used in the framework of a coupled channels formalism for vibrational nuclei. This analysis also included known $s-$ and $p$-wave strength function information. An $\ell$-dependence was not considered in deducing the potential parameters but it was noted that the model overestimated the $p$ - wave strength by approximately $40 \%$.

The present study, while not encompassing a large energy range, is the most extensive high-resolution measurement which extends into the $100 \mathrm{keV}$ region. Very detailed information in the form of individual partial wave contributions is made possible through the high energy resolution of the Oak Ridge Electron Linear Accelerator (ORELA). This partial-wave specific information can be used to determine average nuclear properties for individual $\mathrm{J}^{\pi}$ interactions and to test for $\ell$-dependence of the nuclear potential.

\section{Experimental Measurements}

We have performed transmission measurements by the time-of-flight technique, using neutron pulses from the Oak Ridge Electron Linear Accelerator, at a flight path of $200 \mathrm{~m}$ for a target of ${ }^{122} \mathrm{Sn}$. The $140 \mathrm{MeV}$ electron beam burst width was $15 \mathrm{nsec}$ and the accelerator was pulsed at 800 bursts per second at a power level of approximately $20 \mathrm{~kW}$. The resulting neutron burst has a continuous energy spectrum produced by the photoneutron process in tantalum with subsequent moderation in the $15-\mathrm{cm}$-diameter, berylliumclad, water-filled target housing. Collimation was used to select neutrons from the water-moderated region of the target. The neutron energy resolution function is expected to be a combination in quadrature of the fluctuations in flight-path length and apparent flight time and has been found to have an approximately Gaussian shape with a full width at half maximum, $\mathrm{dE}$, given by

$$
(d E / E)^{2}=(0.16+4 \cdot E) \times 10^{-6},
$$


with $\mathrm{E}$ expressed in $\mathrm{MeV}$. Overlap neutrons were eliminated by a $1-\mathrm{g} / \mathrm{cm}^{2}{ }^{10} \mathrm{~B}$ filter and gamma rays were reduced by a $0.73 \mathrm{~cm}$-thick ${ }^{238} U$ filter, placed $5 \mathrm{~m}$ from the neutron source.

The $29.914 \mathrm{~g}$ sample of ${ }^{122} \mathrm{Sn}$ was $1.59 \mathrm{~cm}$ in diameter, corresponding to a thickness of $13.70 \frac{\mathrm{bams}}{\mathrm{atom}}$. Table I gives the sample enrichment and thickness. We performed additional measurements on a sample of natural tin in order to identify possible impurities in the sample due to the other isotopes of tin. The samples were positioned $9 \mathrm{~m}$ from the neutron target where the neutron beam was collimated to a diameter of 1.4 $\mathrm{cm}$. The samples were cycled into and out of the neutron beam under computer control with a cycle time of approximately $10 \mathrm{~min}$ per sample. A 10-min nun per cycle was also made with no sample in the beam. We used a neutron monitor to compensate for fluctuations in the neutron production rate during the ten day interval and a total of 226 hours of data collection. The five individual runs were each corrected for deadtime and then added to form the final data set. by neutron capture in the water moderator, (2) a constant beam-independent background, (3) a 478-keV gamma ray from the ${ }^{10} B(n, \alpha \gamma)$ reaction resulting from neutrons moderated by the NE110 plastic scintillator and then absorbed by the boron in the Pyrex face of the photomultiplier, and (4) delayed pulses within the photo tube and scintillator. The transmission was computed from the background-corrected sample-in and sample-out ratio, normalized to the corresponding neutron monitor counts.

\section{Results}

As a representative sample of the results for ${ }^{122} \mathrm{Sn}$, Fig. 1 shows the energy range $40-100 \mathrm{keV}$. The uncertainties on the data are shown as vertical lines or are less than the size of the symbols. The solid line represents the R-matrix parametrization of the total cross section discussed in the next section.
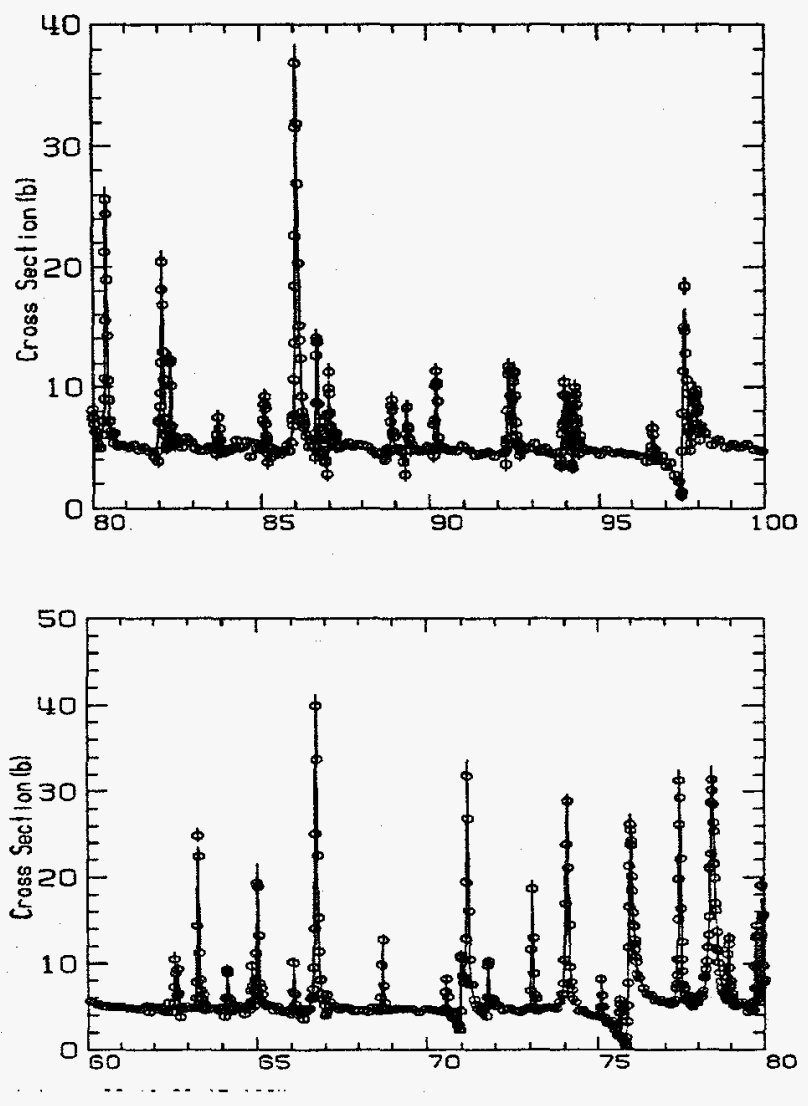

Neutrons were detected by a NE110 proton recoil detector $7.6 \mathrm{~cm}$ in diameter and nominally $2-\mathrm{cm}$ thick. The plastic scintillator was optically coupled to an RCA 8854 photomultiplier tube which was operated in a "selective gating" mode. In this mode, four energy windows are established, the cross-over energies between these windows being 230,630 , and $2020 \mathrm{keV}$. Logic for gating event deadtime in the time digitizer is then determined as follows: a) a single-stop-per-start if an event was identified in time as a gamma flash and occurred in biases 2,3 , or 4 and b) an event occurred in bais 4 at any time. If the event occurred anywhere else, the system operated in a multi-stop-per-start mode with an 1104 nsec deadtime. These separate pulse height spectra facilitated the determination of the backgrounds and the optimization of the signal to background ratio.

We monitored four sources of background during the experiment: (1) $2.2 \mathrm{MeV}$ gamma rays in the beam produced 


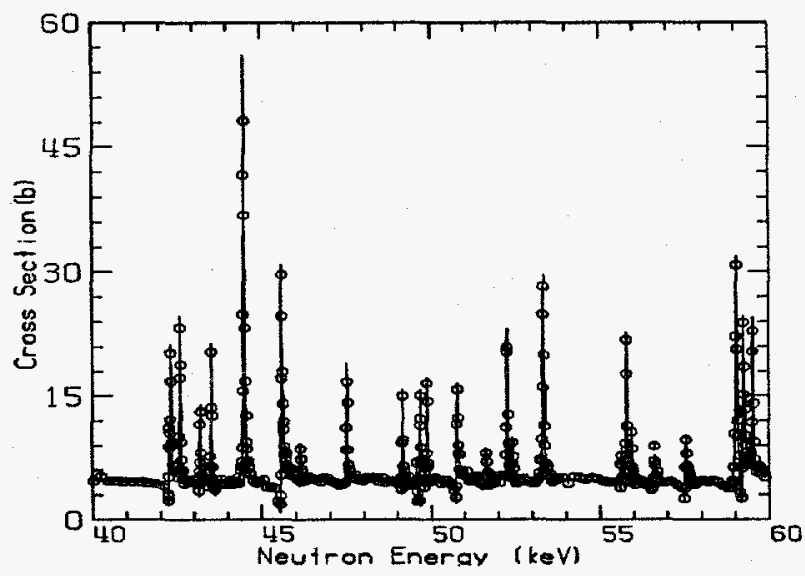

Figure 1. A selected energy region of the total cross section for the ${ }^{122} \mathrm{Sn}+n$ reaction, the circles representing the data and the line the R-matrix fit to the data.

Strong interference patterns are seen at 45,76 , and 97 $\mathrm{keV}$, characteristic of s-wave interaction. Since the target was not a pure sample of ${ }^{122} S n$, the contributions of impurity isotopes are not expected to be insignificant. At these energies, the contribution to the total background cross section is primarily due to the $s$-wave interaction. We have thus included off-resonance s-wave contributions due to ${ }^{116-124} \mathrm{Sn}$, the largest impurity components. At $50-100 \mathrm{keV}$ neutron energies, this contribution to the background cross section amounts to approximately 0.5 barns. Contributions from other partial waves of the impurity components are small at these energies, in comparison to the s-waves. The resonance contributions to the cross sections from the impurity isotopes will be negligible for all but those of very large width.

The early transmission measurements ${ }^{1}$ observed a total of nearly 100 resonances in all the stable isotopes of tin up to a neutron energy of $10 \mathrm{keV}$. In the present case we have extended the energy range to $310 \mathrm{keV}$, and the number of resonances to 347 for just one isotope! From the multilevel resonance analysis we have obtained spin-separated resonance and non-resonant parameters. We have made parity and spin assignments for many of the observed resonances, where earlier results were only able to distinguish $s$ - wave resonances. Besides the normally reported strength functions and level spacings, our $R$ - matrix analysis yields average properties describing the resonances outside the region as deduced from their influence in the region through observed resonance asymmetries. We deduced these parameters for the $s_{1 / 2}, p_{1 / 2}$, and $p_{3 / 2}$ partial wave components. Since the external $R$ - function is related to the real part of the optical model potential, we are able to deduce some of the parameters of this potential, extending previous investigations ${ }^{4}$ of the $\ell$-dependence of the real well depth of the optical model potential.
Table II gives the resonance parameters for $n+{ }^{122} S n$. This isotope is in a region of very low $s$-wave strength function and our results confirm that fact with smaller uncertainty than past measurements. 


\begin{tabular}{|c|c|c|c|c|c|c|c|c|c|c|c|}
\hline $\begin{array}{l}\text { Energy } \\
(\mathrm{keV})\end{array}$ & $\mathbf{J}^{\pi}$ & $\begin{array}{l}g \Gamma_{\mathrm{n}} \\
(\mathrm{eV})\end{array}$ & $\begin{array}{r}g \gamma_{\mathrm{n}}^{2} \\
(\mathrm{eV})\end{array}$ & $\begin{array}{c}\text { Energy } \\
\text { (keV) }\end{array}$ & $J^{\pi}$ & $\begin{array}{l}g \Gamma_{\mathrm{n}} \\
(\mathrm{eV})\end{array}$ & $\begin{array}{l}g \gamma_{\mathrm{n}}^{2} \\
(\mathrm{eV})\end{array}$ & $\begin{array}{c}\text { Energy } \\
(\mathrm{keV})\end{array}$ & $J^{n}$ & $\begin{array}{r}\mathrm{g} \Gamma_{\mathrm{n}} \\
(\mathrm{eV})\end{array}$ & $\begin{array}{r}\mathrm{g} \gamma_{\mathrm{n}}^{2} \\
(\mathrm{eV})\end{array}$ \\
\hline 13.231 & $\left(3 / 2^{-}\right)$ & 5 & 472 & 53.332 & $3 / 2^{-}$ & 37 & 436 & 97.545 & $1 / 2^{+}$ & 61 & 62 \\
\hline 13.818 & $\left(3 / 2^{-}\right)$ & 5 & 429 & 55.628 & $\left(1 / 2^{-}\right)$ & 3 & 35 & 97.816 & $1 / 2^{+}$ & 14 & 14 \\
\hline 15.061 . & $\left(3 / 2^{-}\right)$ & 3 & 209 & 55.783 & $\left(3 / 2^{-}\right)$ & 23 & 261 & 101.854 & $1 / 2^{-}$ & 183 & 914 \\
\hline 15.079 & $\left(3 / 2^{-}\right)$ & 4 & 310 & 55.934 & $\left(3 / 2^{-}\right)$ & 8 & 85 & 102.719 & $3 / 2^{-}$ & 33 & 163 \\
\hline 15.866 & $\left(3 / 2^{-}\right)$ & 4 & 248 & 56.591 & $1 / 2^{+}$ & 5 & 6 & 104.200 & $\left(1 / 2^{-}\right)$ & 22 & 107 \\
\hline 17.150 & $\left(3 / 2^{-}\right)$ & 3 & 175 & 57.534 & $1 / 2^{+}$ & 6 & 8 & 104.475 & $1 / 2^{+}$ & 10 & 10 \\
\hline 18.104 & $\left(1 / 2^{-}\right)$ & 4 & 195 & 59.038 & $1 / 2^{-}$ & 41 & 426 & 105.156 & $\left(1 / 2^{-}\right)$ & 12 & 56 \\
\hline 18.962 & $1 / 2^{+}$ & 9 & 21 & 59.122 & $\left(3 / 2^{-}\right)$ & 9 & 88 & 106.892 & $1 / 2^{+}$ & 23 & 22 \\
\hline 19.418 & $\left(1 / 2^{-}\right)$ & 3 & 152 & 59.238 & $1 / 2^{+}$ & 26 & 34 & 107.026 & $(1 / 2)$ & 25 & 119 \\
\hline 20.634 & $1 / 2^{+}$ & 4 & 10 & 59.524 & $\left(3 / 2^{\circ}\right)$ & 28 & 288 & 108.262 & $\left(3 / 2^{-}\right)$ & 56 & 259 \\
\hline 22.136 & $\left(3 / 2^{-}\right)$ & 4 & 161 & 62.626 & $\left(1 / 2^{-}\right)$ & 8 & 73 & 108.471 & $1 / 2^{+}$ & 4 & 4 \\
\hline 22.907 & $\left(1 / 2^{-}\right)$ & 11 & 424 & 62.705 & $\left(3 / 2^{\circ}\right)$ & 6 & 56 & 108.624 & $\left(1 / 2^{-}\right)$ & 77 & 354 \\
\hline 24.125 & $\left(1 / 2^{-}\right)$ & 6 & 231 & 63.299 & $3 / 2^{-}$ & 39 & 369 & 108.982 & $\left(1 / 2^{-}\right)$ & 43 & 196 \\
\hline 24.510 & $\left(3 / 2^{-}\right)$ & 6 & 202 & 64.140 & $\left(3 / 2^{\circ}\right)$ & 9 & 85 & 110.786 & $\left(1 / 2^{-}\right)$ & 41 & 182 \\
\hline 26.278 & $\left(1 / 2^{-}\right)$ & 4 & 116 & 64.851 & $\left(3 / 2^{-}\right)$ & 9 & 80 & 113.549 & $\left(3 / 2^{-}\right)$ & 97 & 422 \\
\hline 27.102 & $1 / 2^{+}$ & 36 & 69 & 65.021 & $1 / 2^{-}$ & 29 & 262 & 114.058 & $\left(3 / 2^{\prime}\right)$ & 39 & 170 \\
\hline 27.118 & $\left(1 / 2^{-}\right)$ & 4 & 116 & 66.073 & $\left(3 / 2^{-}\right)$ & 8 & 75 & 114.444 & $3 / 2^{-}$ & 60 & 258 \\
\hline 28.281 & $3 / 2^{-}$ & 17 & 497 & 66.735 & $3 / 2^{-}$ & 88 & 772 & 114.961 & $3 / 2^{-}$ & 144 & 612 \\
\hline 28.423 & $1 / 2^{-}$ & 12 & 335 & 68.730 & $\left(3 / 2^{-}\right)$ & 16 & 132 & 115.498 & $1 / 2^{+}$ & 13 & 13 \\
\hline 28.889 & $\left(1 / 2^{-}\right)$ & 3 & 76 & 70.576 & $\left(3 / 2^{-}\right)$ & 8 & 69 & 116.707 & $\left(3 / 2^{-}\right)$ & 19 & 79 \\
\hline 31.591 & $1 / 2^{+}$ & 13 & 23 & 70.987 & $1 / 2^{+}$ & 13 & 16 & 117.372 & $\left(3 / 2^{-}\right)$ & 74 & 306 \\
\hline 31.869 & $1 / 2^{-}$ & 11 & 281 & 71.182 & $3 / 2^{-}$ & 70 & 564 & 118.507 & $\left(3 / 2^{-}\right)$ & 19 & 77 \\
\hline 32.337 & $1 / 2^{-}$ & 26 & 620 & 71.765 & $1 / 2^{+}$ & 9 & 10 & 118.509 & $1 / 2^{+}$ & 2 & 2 \\
\hline 33.288 & $1 / 2^{+}$ & 2 & 4 & 73.065 & $3 / 2$ & 33 & 258 & 118.778 & $\left(3 / 2^{-}\right)$ & 45 & 185 \\
\hline 34.453 & $\left(3 / 2^{-}\right)$ & 7 & 145 & 74.072 & $1 / 2$ & 75 & 568 & 120.389 & $\left(3 / 2^{-}\right)$ & 44 & 177 \\
\hline 34.994 & $\left(1 / 2^{-}\right)$ & 8 & 179 & 75.093 & $\left(3 / 2^{-}\right)$ & 10 & 75 & 121.567 & $\left(3 / 2^{-}\right)$ & 18 & 70 \\
\hline 35.256 & $\left(1 / 2^{-}\right)$ & 2 & 38 & 75.651 & $(3 / 2)$ & 8 & 62 & 121.911 & $3 / 2^{-}$ & 71 & 283 \\
\hline 35.393 & $1 / 2^{+}$ & 1 & 1 & 75.942 & $1 / 2^{+}$ & 87 & 101 & 122.575 & $3 / 2^{-}$ & 119 & 467 \\
\hline 35.440 & $1 / 2^{-}$ & 27 & 567 & 77.436 & $3 / 2$ & 72 & 514 & 123.561 & $\left(3 / 2^{-}\right)$ & 57 & 223 \\
\hline 35.568 & $1 / 2^{+}$ & 8 & 13 & 78.426 & $1 / 2$ & 151 & 1064 & 124.058 & $1 / 2^{+}$ & 25 & 23 \\
\hline 36.863 & $\left(1 / 2^{-}\right)$ & 5 & 106 & 78.925 & $\left(3 / 2^{-}\right)$ & 18 & 127 & 125.687 & $\left(1 / 2^{-}\right)$ & 52 & 199 \\
\hline 37.387 & $\left(3 / 2^{-}\right)$ & 9 & 180 & 79.739 & $\left(1 / 2^{-}\right)$ & 19 & 134 & 126.111 & $\left(1 / 2^{-}\right)$ & 11 & 41 \\
\hline 38.193 & $\left(1 / 2^{-}\right)$ & 1 & 25 & 79.747 & $1 / 2^{+}$ & 5 & 6 & 126.730 & $\left(1 / 2^{-}\right)$ & 24 & 89 \\
\hline 38.584 & $1 / 2^{-}$ & 50 & 945 & 79.919 & $1 / 2^{-}$ & 27 & 188 & 127.488 & $3 / 2^{-}$ & 210 & 784 \\
\hline 42.265 & $1 / 2^{+}$ & 4 & 6 & 80.390 & $3 / 2$ & 59 & 400 & 127.530 & $1 / 2^{+}$ & 7 & 7 \\
\hline 42.318 & $3 / 2^{-}$ & 12 & 191 & 81.973 & $1 / 2^{+}$ & 5 & 6 & 129.509 & $\left(3 / 2^{-}\right)$ & 59 & 215 \\
\hline 42.603 & $\left(1 / 2^{-}\right)$ & 13 & 211 & 82.088 & $3 / 2^{-}$ & 39 & 261 & 130.596 & $\left(3 / 2^{-}\right)$ & 53 & 192 \\
\hline 43.197 & $\left(3 / 2^{-}\right)$ & 7 & 115 & 82.327 & $3 / 2^{-}$ & 19 & 128 & 132.445 & $1 / 2^{-}$ & 121 & 431 \\
\hline 43.520 & $(3 / 2)$ & 12 & 191 & 83.743 & $\left(3 / 2^{-}\right)$ & 3 & 19 & 133.280 & $\left(3 / 2^{-}\right)$ & 28 & 99 \\
\hline 44.497 & $3 / 2$ & 50 & 764 & 85.091 & $\left(3 / 2^{-}\right)$ & 13 & 81 & 134.853 & $\left(1 / 2^{-}\right)$ & 60 & 208 \\
\hline 45.594 & $1 / 2^{+}$ & 23 & 35 & 86.054 & $3 / 2^{-}$ & 122 & 757 & 135.480 & $\left(1 / 2^{-}\right)$ & 8 & 26 \\
\hline 46.151 & $\left(3 / 2^{-}\right)$ & 3 & 44 & 86.621 & $\left(3 / 2^{\circ}\right)$ & 27 & 168 & 136.158 & $\left(1 / 2^{-}\right)$ & 16 & 56 \\
\hline 47.471 & $1 / 2^{+}$ & 2 & 4 & 86.950 & $1 / 2^{+}$ & 14 & 15 & 137.624 & $\left(1 / 2^{-}\right)$ & 37 & 125 \\
\hline 47.510 & $1 / 2^{-}$ & 8 & 118 & 88.892 & $\left(3 / 2^{-}\right)$ & 14 & 81 & 137.948 & $3 / 2^{\circ}$ & 254 & 860 \\
\hline 49.154 & $\left(3 / 2^{-}\right)$ & 10 & 135 & 89.319 & $1 / 2^{+}$ & 8 & 8 & 140.181 & $\left(1 / 2^{-}\right)$ & 21 & 71 \\
\hline 49.567 & $\left(3 / 2^{-}\right)$ & 4 & 50 & 90.198 & $\left(3 / 2^{-}\right)$ & 20 & 119 & 141.351 & $\left(1 / 2^{-}\right)$ & 20 & 65 \\
\hline 49.666 & $1 / 2^{+}$ & 10 & 14 & 92.348 & $\left(3 / 2^{-}\right)$ & 30 & 169 & 141.776 & $1 / 2^{+}$ & 3 & 3 \\
\hline 49.885 & $\left(3 / 2^{-}\right)$ & 12 & 157 & 92.488 & $\left(3 / 2^{-}\right)$ & 25 & 142 & 141.882 & $\left(1 / 2^{-}\right)$ & 53 & 174 \\
\hline 50.765 & $1 / 2^{+}$ & 11 & 16 & 93.964 & $\left(1 / 2^{-}\right)$ & 19 & 107 & 143.809 & $\left(1 / 2^{-}\right)$ & 37 & 120 \\
\hline 51.629 & $1 / 2^{+}$ & 2 & 3 & 94.098 & $\left(1 / 2^{-}\right)$ & 14 & 79 & 144.443 & $\left(3 / 2^{-}\right)$ & 17 & 56 \\
\hline 52.265 & $\left(3 / 2^{-}\right)$ & 23 & 281 & 94.243 & $1 / 2^{+}$ & 16 & 16 & 145.387 & $1 / 2^{+}$ & 27 & 23 \\
\hline 52.424 & $\left(1 / 2^{*}\right)$ & 4 & 46 & 96.629 & $\left(3 / 2^{-}\right)$ & 8 & 43 & 145.412 & $\left(1 / 2^{-}\right)$ & 103 & 325 \\
\hline
\end{tabular}




\begin{tabular}{|c|c|c|c|c|c|c|c|c|c|c|c|}
\hline $\begin{array}{l}\text { Energy } \\
(\mathrm{keV})\end{array}$ & $\mathrm{J}^{\pi}$ & $\begin{array}{l}g \Gamma_{\mathrm{n}} \\
(\mathrm{eV})\end{array}$ & $\begin{array}{r}\mathrm{g}_{\mathrm{n}}^{2} \\
(\mathrm{eV})\end{array}$ & $\begin{array}{c}\text { Energy } \\
(\mathrm{keV})\end{array}$ & $J^{\pi}$ & $\begin{array}{l}\mathrm{g} \Gamma_{\mathrm{n}} \\
(\mathrm{eV})\end{array}$ & $\begin{array}{l}\mathrm{g} \gamma_{\mathrm{n}}^{2} \\
(\mathrm{eV})\end{array}$ & $\begin{array}{c}\text { Energy } \\
(\mathrm{keV})\end{array}$ & $J^{\pi}$ & $\begin{array}{c}\mathrm{g} \Gamma_{\mathrm{n}} \\
(\mathrm{eV})\end{array}$ & $\begin{array}{r}\mathrm{g} \gamma_{\mathrm{n}}^{2} \\
(\mathrm{eV})\end{array}$ \\
\hline 146.166 & $(1 / 2)$ & 48 & 150 & 180.337 & $1 / 2^{-}$ & 76 & 185 & 220.787 & $3 / 2$ & 242 & 466 \\
\hline 147.506 & $(1 / 2)$ & 75 & 234 & 180.713 & $3 / 2^{-}$ & 98 & 238 & 221.422 & $3 / 2$ & 91 & 175 \\
\hline 149.138 & $(3 / 2)$ & 46 & 141 & 181.122 & $1 / 2^{+}$ & 4 & 3 & 221.498 & $1 / 2^{+}$ & 26 & 17 \\
\hline 150.323 & $1 / 2^{+}$ & 5 & 4 & 181.233 & $3 / 2$ & 188 & 457 & 222.382 & $\left(1 / 2^{-}\right)$ & 25 & 47 \\
\hline 150.458 & $\left(1 / 2^{-}\right)$ & 60 & 183 & 182.240 & $3 / 2^{-}$ & 104 & 251 & 223.224 & $\left(3 / 2^{-}\right)$ & 81 & 154 \\
\hline 151.228 & $\left(1 / 2^{-}\right)$ & 57 & 174 & 183.420 & $1 / 2^{-}$ & 249 & 596 & 224.413 & $\left(1 / 2^{-}\right)$ & 47 & 88 \\
\hline 151.566 & $1 / 2^{+}$ & 4 & 3 & 183.830 & $\left(1 / 2^{-}\right)$ & 17 & 40 & 226.508 & $(1 / 2)$ & 21 & 40 \\
\hline 152.834 & $\left(3 / 2^{-}\right)$ & 43 & 128 & 185.302 & $\left(1 / 2^{-}\right)$ & 34 & 80 & 226.976 & $\left(1 / 2^{-}\right)$ & 48 & 90 \\
\hline 153.100 & $1 / 2^{+}$ & 8 & 6 & 185.806 & $\left(3 / 2^{-}\right)$ & 9 & 21 & 229.202 & $1 / 2^{-}$ & 110 & 203 \\
\hline 154.060 & $(3 / 2)$ & 29 & 87 & 186.343 & $3 / 2^{-}$ & 41 & 96 & 230.978 & $1 / 2^{-}$ & 75 & 137 \\
\hline 154.285 & $\left(3 / 2^{-}\right)$ & 10 & 31 & 186.748 & $1 / 2^{-}$ & 160 & 375 & 231.461 & $\left(1 / 2^{-}\right)$ & 45 & 82 \\
\hline 155.139 & $\left(3 / 2^{-}\right)$ & 49 & 144 & 187.329 & $\left(3 / 2^{-}\right)$ & 41 & 96 & 231.842 & $\left(3 / 2^{-}\right)$ & 60 & 110 \\
\hline 156.040 & $1 / 2^{+}$ & 46 & 37 & 187.921 & $\left(1 / 2^{-}\right)$ & 8 & 20 & 232.460 & $\left(3 / 2^{-}\right)$ & 12 & 22 \\
\hline 156.077 & $\left(3 / 2^{\circ}\right)$ & 50 & 145 & 188.177 & $1 / 2^{+}$ & 23 & 17 & 232.861 & $\left(1 / 2^{-}\right)$ & 38 & 69 \\
\hline 156.774 & $\left(1 / 2^{\circ}\right)$ & 28 & 80 & 190.112 & $1 / 2$ & 53 & 121 & 233.454 & $\left(3 / 2^{-}\right)$ & 41 & 74 \\
\hline 156.966 & $\left(1 / 2^{-}\right)$ & 59 & 171 & 191.300 & $3 / 2^{-}$ & 220 & 500 & 233.700 & $1 / 2^{+}$ & 15 & 10 \\
\hline 158.777 & $(1 / 2)$ & 55 & 156 & 191.886 & $1 / 2^{+}$ & 12 & 9 & 234.447 & $\left(1 / 2^{-}\right)$ & 65 & 117 \\
\hline 160.778 & $(3 / 2)$ & 34 & 96 & 192.505 & $\left(3 / 2^{-}\right)$ & 9 & 20 & 235.783 & $3 / 2$ & 200 & 358 \\
\hline 161.148 & $\left(3 / 2^{\prime}\right)$ & 39 & 109 & 193.207 & $\left(1 / 2^{-}\right)$ & 17 & 37 & 237.539 & $\left(1 / 2^{-}\right)$ & 42 & 74 \\
\hline 161.176 & $1 / 2^{+}$ & 9 & 7 & 193.976 & $\left(1 / 2^{-}\right)$ & 17 & 37 & 239.753 & $\left(3 / 2^{-}\right)$ & 77 & 136 \\
\hline 161.416 & $\left(3 / 2^{-}\right)$ & 24 & 67 & 195.836 & $\left(3 / 2^{-}\right)$ & 76 & 169 & 240.218 & $\left(1 / 2^{-}\right)$ & 22 & 39 \\
\hline 161.818 & $1 / 2^{+}$ & 7 & 6 & 196.099 & $\left(1 / 2^{-}\right)$ & 21 & 47 & 240.821 & $\left(1 / 2^{-}\right)$ & 28 & 49 \\
\hline 162.931 & $\left(3 / 2^{-}\right)$ & 85 & 235 & 197.349 & $\left(3 / 2^{-}\right)$ & 35 & 77 & 241.106 & $1 / 2^{+}$ & 9 & 6 \\
\hline 163.222 & $\left(3 / 2^{-}\right)$ & 5 & 15 & 198.087 & $\left(1 / 2^{-}\right)$ & 9 & 19 & 241.334 & $1 / 2$ & 268 & 466 \\
\hline 163.434 & $1 / 2^{+}$ & 20 & 16 & 198.575 & $3 / 2^{-}$ & 455 & 992 & 241.991 & $1 / 2^{+}$ & 14 & 9 \\
\hline 163.697 & $\left(1 / 2^{\prime}\right)$ & 7 & 18 & 199.449 & $\left(1 / 2^{-}\right)$ & 20 & 43 & 242.960 & $\left(1 / 2^{-}\right)$ & 31 & 53 \\
\hline 164.193 & $1 / 2^{+}$ & 13 & 10 & 200.364 & $3 / 2^{-}$ & 185 & 399 & 243.788 & $\left(1 / 2^{-}\right)$ & 37 & 64 \\
\hline 164.310 & $(3 / 2)$ & 90 & 247 & 201.623 & $3 / 2$ & 151 & 324 & 244.207 & $\left(3 / 2^{-}\right)$ & 62 & 106 \\
\hline 165.150 & $1 / 2^{+}$ & 15 & 12 & 201.864 & $\left(1 / 2^{-}\right)$ & 40 & 85 & 245.304 & $\left(1 / 2^{\prime}\right)$ & 125 & 214 \\
\hline 165.178 & $\left(3 / 2^{-}\right)$ & 28 & 77 & 202.463 & $1 / 2^{+}$ & 20 & 14 & 245.756 & $\left(3 / 2^{-}\right)$ & 86 & 146 \\
\hline 165.753 & $\left(1 / 2^{-}\right)$ & 21 & 58 & 203.219 & $\left(1 / 2^{-}\right)$ & 70 & 149 & 246.079 & $\left(1 / 2^{-}\right)$ & 160 & 272 \\
\hline 166.652 & $\left(3 / 2^{-}\right)$ & 110 & 294 & 205.197 & $\left(1 / 2^{-}\right)$ & 71 & 148 & 249.857 & $1 / 2^{-}$ & 702 & 1175 \\
\hline 166.726 & $1 / 2^{+}$ & 31 & 24 & 205.615 & $\left(3 / 2^{-}\right)$ & 61 & 128 & 250.594 & $3 / 2^{-}$ & 124 & 207 \\
\hline 167.025 & $\left(3 / 2^{-}\right)$ & 107 & 286 & 205.989 & $\left(3 / 2^{-}\right)$ & 15 & 32 & 252.499 & $3 / 2^{\circ}$ & 186 & 308 \\
\hline 167.426 & $\left(3 / 2^{-}\right)$ & 70 & 187 & 207.761 & $1 / 2^{+}$ & 37 & 26 & 252.816 & $\left(3 / 2^{-}\right)$ & 70 & 115 \\
\hline 168.100 & $1 / 2^{+}$ & 12 & 9 & 208.022 & $1 / 2^{-}$ & 125 & 258 & 253.315 & $\left(3 / 2^{-}\right)$ & 82 & 136 \\
\hline 169.405 & $3 / 2^{-}$ & 60 & 158 & 208.976 & $\left(3 / 2^{-}\right)$ & 59 & 122 & 254.118 & $\left(1 / 2^{-}\right)$ & 124 & 204 \\
\hline 170.035 & $1 / 2^{+}$ & 6 & 5 & 209.468 & $\left(1 / 2^{-}\right)$ & 24 & 49 & 255.212 & $\left(3 / 2^{-}\right)$ & 82 & 134 \\
\hline 170.110 & $3 / 2^{-}$ & 40 & 104 & 210.638 & $3 / 2^{-}$ & 36 & 73 & 255.717 & $\left(3 / 2^{-}\right)$ & 91 & 149 \\
\hline 170.281 & $\left(1 / 2^{-}\right)$ & 9 & 24 & 211.789 & $1 / 2^{-}$ & 287 & 580 & 256.158 & $3 / 2^{-}$ & 177 & 289 \\
\hline 171.739 & $\left(3 / 2^{-}\right)$ & 17 & 44 & 212.273 & $\left(3 / 2^{-}\right)$ & 14 & 28 & 257.426 & $\left(3 / 2^{-}\right)$ & 113 & 183 \\
\hline 173.224 & $1 / 2^{+}$ & 16 & 12 & 213.129 & $\left(3 / 2^{-}\right)$ & 57 & 115 & 257.919 & $\left(3 / 2^{-}\right)$ & 43 & 70 \\
\hline 173.366 & $\left(1 / 2^{-}\right)$ & 16 & 40 & 213.489 & $\left(1 / 2^{-}\right)$ & 26 & 52 & 260.065 & $\left(3 / 2^{-}\right)$ & 50 & 80 \\
\hline 174.472 & $\left(1 / 2^{-}\right)$ & 40 & 102 & 213.929 & $\left(1 / 2^{-}\right)$ & 23 & 47 & 260.638 & $3 / 2^{\prime}$ & 221 & 354 \\
\hline 174.687 & $(3 / 2)$ & 5 & 13 & 214.203 & $3 / 2$ & 172 & 342 & 261.642 & $\left(3 / 2^{\circ}\right)$ & 25 & 39 \\
\hline 176.164 & $\left(3 / 2^{-}\right)$ & 58 & 145 & 215.588 & $3 / 2$ & 207 & 411 & 262.787 & $3 / 2^{\circ}$ & 260 & 412 \\
\hline 176.435 & $1 / 2^{+}$ & 53 & 40 & 216.415 & $\left(1 / 2^{-}\right)$ & 17 & 33 & 263.942 & $\left(1 / 2^{-}\right)$ & 167 & 263 \\
\hline 176.707 & $\left(1 / 2^{-}\right)$ & 26 & 64 & 217.646 & $1 / 2^{+}$ & 28 & 19 & 264.240 & $\left(3 / 2^{-}\right)$ & 78 & 122 \\
\hline 178.471 & $\left(1 / 2^{-}\right)$ & 20 & 49 & 217.947 & $3 / 2$ & 99 & 193 & 265.150 & $1 / 2^{+}$ & 16 & 10 \\
\hline 179.076 & $\left(1 / 2^{-}\right)$ & 116 & 286 & 218.795 & $3 / 2$ & 133 & 259 & 265.409 & $\left(3 / 2^{-}\right)$ & 187 & 293 \\
\hline 179.704 & $1 / 2^{+}$ & 23 & 18 & 218.882 & $1 / 2^{+}$ & 33 & 22 & 265.700 & $\left(1 / 2^{-}\right)$ & 40 & 63 \\
\hline 179.875 & $\left(1 / 2^{-}\right)$ & 15 & 36 & 219.415 & $\left(3 / 2^{-}\right)$ & 17 & 33 & 266.592 & $\left(3 / 2^{\prime}\right)$ & 85 & 133 \\
\hline
\end{tabular}




$\begin{array}{ccrr}\begin{array}{c}\text { Energy } \\ (\mathrm{keV})\end{array} & \mathrm{J}^{\mathrm{T}} & \begin{array}{r}\mathrm{g} \Gamma_{\mathrm{n}} \\ (\mathrm{eV})\end{array} & \begin{array}{r}\mathrm{g} \gamma_{\mathrm{n}}^{2} \\ (\mathrm{eV})\end{array} \\ 268.144 & \left(3 / 2^{-}\right) & 107 & 166 \\ 268.365 & \left(1 / 2^{-}\right) & 48 & 74 \\ 271.975 & 1 / 2^{-} & 225 & 342 \\ 272.873 & 1 / 2^{-} & 77 & 116 \\ 274.544 & \left(1 / 2^{-}\right) & 33 & 50 \\ 275.307 & 1 / 2^{+} & 37 & 22 \\ 276.031 & \left(1 / 2^{-}\right) & 153 & 230 \\ 276.839 & \left(3 / 2^{-}\right) & 87 & 129 \\ 277.591 & \left(3 / 2^{-}\right) & 70 & 104 \\ 278.933 & \left(1 / 2^{-}\right) & 21 & 32 \\ 280.195 & \left(1 / 2^{-}\right) & 105 & 155 \\ 281.026 & \left(1 / 2^{-}\right) & 172 & 253 \\ 281.967 & \left(1 / 2^{-}\right) & 76 & 112 \\ 282.139 & 1 / 2^{+} & 27 & 16 \\ 284.670 & 3 / 2^{-} & 190 & 276 \\ 286.174 & \left(1 / 2^{-}\right) & 58 & 84 \\ 286.590 & 3 / 2^{-} & 340 & 489 \\ 287.061 & \left(3 / 2^{-}\right) & 81 & 116 \\ 287.800 & 1 / 2^{+} & 35 & 21 \\ 287.996 & \left(3 / 2^{-}\right) & 88 & 126 \\ 289.026 & \left(3 / 2^{-}\right) & 61 & 88 \\ 289.620 & \left(3 / 2^{-}\right) & 140 & 200 \\ 290.338 & \left(3 / 2^{-}\right) & 82 & 116 \\ 292.261 & \left(3 / 2^{-}\right) & 51 & 72 \\ 293.641 & \left(3 / 2^{-}\right) & 115 & 161 \\ 295.730 & \left(3 / 2^{-}\right) & 53 & 73 \\ 296.106 & \left(1 / 2^{-}\right) & 37 & 51 \\ 298.264 & \left(1 / 2^{-}\right) & 146 & 202 \\ 298.620 & \left(3 / 2^{-}\right) & 118 & 163 \\ 298.977 & \left(1 / 2^{-}\right) & 97 & 133 \\ 301.763 & \left(3 / 2^{-}\right) & 192 & 262 \\ 305.601 & \left(1 / 2^{-}\right) & 350 & 470 \\ 308.781 & \left(1 / 2^{-}\right) & 81 & 107 \\ 309.073 & \left(1 / 2^{-}\right) & 206 & 273 \\ & & & \end{array}$

\section{R-matrix Analysis}

The total cross section is expressible in terms of a hard sphere phase shift, $\phi$, and an R-function which depends primarily upon the energy and width parameters of all the individual resonances and an external R-function as,

$$
R_{\ell J}(E)=\sum_{\lambda=1}^{N} \frac{\gamma_{\lambda \ell J}^{2}}{E_{\lambda \ell J}-E}+R_{\ell J}^{e x t}(E)
$$

where $\gamma_{U}^{2}$ and $E_{U \nu}$ are free parameters representing the reduced width and energy of the $\lambda_{t h}$ resonance, with the $\gamma_{t}^{2}$ related to the neutron widths, $\Gamma_{n}$, by the relation,

$$
\gamma_{\ell J}^{2}=\frac{\Gamma_{r \ell J}}{2 P_{\ell}}
$$

The external R-function, in combination with $\phi_{\ell}$, influences both the off-resonance cross section and the interference pattern of the resonances. We write this part of the R-function as

$$
R_{\ell J}^{e x t}(E)=\widetilde{R}-\widetilde{s} \cdot \ln \left[\frac{E_{u p}-E}{E-E_{b}}\right]
$$

with the smooth function, $\widetilde{R}$, parametrized as

$$
\widetilde{R}_{\ell J}(E)=\alpha_{\ell J}+\beta_{\ell J} \cdot E,
$$

and $\alpha_{\ell J}$ and $\beta_{\ell J}$ being free parameters. This parametrization provides a simple procedure for averaging of the scattering function for comparison to the optical model. At low energy the $s$ - wave smooth $R$ - function is related to the potential scattering length, $R_{o}^{\prime}$, by

$$
R_{o}^{\prime}=a_{c}\left[1-\widetilde{R}_{o}(E=0)\right]
$$

where $\widetilde{R}_{o}$ is evaluated for $E=0$. The final $R$ - matrix parameters which best described the observed transmissions were determined by solving Bayes' equations using the $R$ - matrix code SAMMY 5 .

The $s$ - wave resonances are easily distinguished by their characteristic asymmetry. We have assumed all other resonances to be due to p-wave interaction because of the small probability of $d$-wave interaction in this energy range. We used an iterative procedure to determine most of the $\mathrm{J}^{\pi}$ assignments, requiring the final assignment to provide a good description of the asymmetry patterns. Resonances whose spin assignments were uncertain are indicated in the table of resonance parameters with parentheses.

\section{Average Properties}

The average properties of interest in the analysis of neutron total cross section data are the strength functions, the level spacings and the external $R$-functions. These are related to interest in the statistical and optical models. We will treat each of these properties in turn.

\section{A. Strength Functions}

The $R$ - matrix strength functions, $\widetilde{s}$, are represented by the slopes of the plot of the cumulative reduced neutron width (See eqn. 2) versus energy distribution, presented in Fig. 2 for the $s$ - and $p$-wave resonances. In the case where the $J$-value samples are not pure, one considers the strength for a given $\ell-$ value. In the case of $p$ waves, for example, the plot has the cumulative reduced neutron width replaced by the expression

$$
g_{\mathrm{J}} \gamma_{\lambda}^{2} /(2 \ell+1)
$$


where $\gamma_{\lambda}^{2}$ is the reduced neutron width of the $\lambda_{t h}$ resonance and $\ell$ is the orbital angular momentum quantum number. The slope of this curve gives the $p$ - wave strength. The resulting values for $\widetilde{s}$ differ by a factor of 15 .

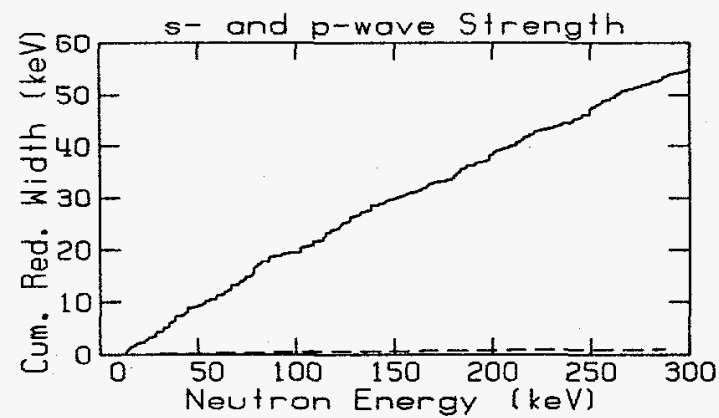

Figure 2. $s$ - and $p$-wave strength. The cumulative values for the $p$ - waves (solid histogram) represent that of Eqn. 6 The slopes of the histograms give the strengths. The sum for $s$-waves is only $\approx 1 \mathrm{keV}$.

From the plots we can see that the strength functions are constant over the energy region, since a single line passed through the histograms would sufficiently describe the trend over a limited energy range. From the relation

$$
\widetilde{s}_{\ell}=\frac{\sum_{\lambda J} g_{\mathrm{J}} \gamma_{\lambda}^{2} /(2 \ell+1)}{\Delta E_{\ell}},
$$

the total $p$-wave strength is calculated to be $(2.0 \pm 0.2) \times 10^{-4}$. This value is in excellent agreement with trends in this mass region. The value for the $s$-wave strength function, $(0.12 \pm 0.02) \times 10^{-4}$, is an order of magnitude lower than that for the $p$-waves but consistent with results for other isotopes of tin and with theoretical investigations of nuclear structure in this mass region.

In Fig. 3 we present the cumulative reduced neutron widths versus neutron energy for the individual $p$-wave components. There is an element of uncertainty in the apportionment of small resonances among the two spin states. The apportionment for these histograms was made on the basis of calculated $\chi^{2}$ values, when fitting with each possibility, and the character of the resulting fits. It is seen that the strength for the $p_{1 / 2}$ component exceeds that of the $p_{3 / 2}$ by $30 \%$ despite the fact that the level density for the latter is greater. Such enhancement has been reported in similar studies on ${ }^{86} K r^{6}$.

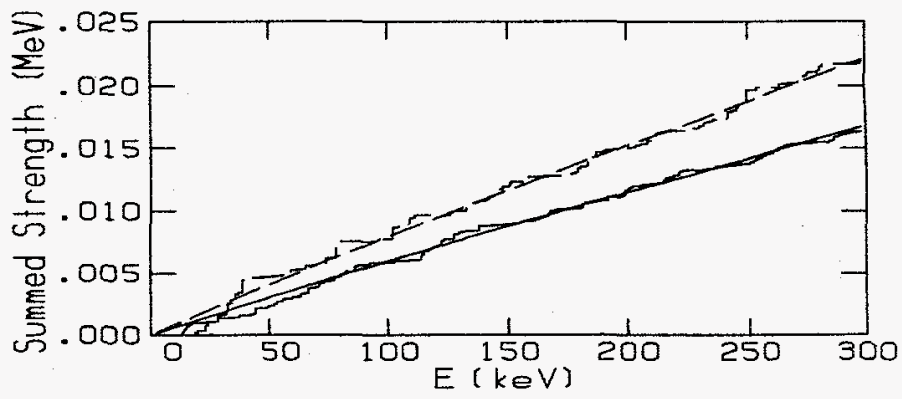

Figure 3. Strength functions for $\boldsymbol{p}$ - wave components. The dashed histogram is for the $p_{1 / 2}$ partial wave. The slopes of these histograms give the corresponding strength functions. The solid lines represent optical model fits, discussed in Section VI.

We have established lower limits for the individual $p_{1 / 2}$ and $p_{3 / 2}$ strength functions and level spacings by including only resonances whose peak height, resonance asymmetry or close proximity to a large resonance made the $J^{\pi}$ assignment definite. The upper limit, for each partial wave, comes from including all resonances of indefinite $\mathrm{J}^{\pi}$ in each of the spin groups, separately. Each of the $p$-wave spin groups constructed in this manner have $70-80 \%$ of the resonances indefinite with approximately $50 \%$ of the resulting strength attributable to these uncertain resonances. Average resonance parameters, their statistical uncertainties, and their upper and lower limits are presented in Table III, in conventional units. The $R$ - matrix strength functions, $\widetilde{\mathbf{s}}$, will be discussed below in connection with the external $R$-functions. The significance of this work is the reduced uncertainty on the $s$ - and $p$ - wave strength functions and the lower and upper limits placed on the component $p$-wave strength functions and level spacings.

Table III. Average Resonance Parameters for ${ }^{122} S n+n$ :

\begin{tabular}{ccc}
$J^{\pi}$ & $S_{\mathrm{J}}^{\ell}\left(\times 10^{+4}\right)$ & $D(\mathrm{keV})$ \\
\hline$s$ & $0.12(2)^{\dagger}$ & $4.1(3)$ \\
$p$ & $2.0(2)$ & $1.06(3)$ \\
$p_{1 / 2}$ & $2.4(3)_{1.4}^{4.1}$ & $2.4(1)_{1.3}^{10.2}$ \\
$p_{3 / 2}$ & $1.8(2)_{1.0}^{2.4}$ & $1.8(1)_{1.1}^{5.9}$ \\
\hline
\end{tabular}

In our notation, 0.12 (2) is equivalent to $0.12 \pm 0.02$ and 2.4(3).4. indicates that upper and lower limits are 4.1 and 1.4, respectively as discussed in the text. The numbers in parentheses represent statistical uncertainties

\section{B. Level Spacings}

From the plot of the cumulative number of s-wave resonances (--) in Figure 4, we see that a total of 66 resonances were observed up to an energy of $310 \mathrm{keV}$. It would appear that up to approximately $240 \mathrm{keV}$, few resonances were missed. Above that energy the increased probability of $p$-wave interaction, coupled with the 
extremely low $s$ - wave strength, results in missed $s$ - wave resonances. We have not attempted a correction for the missed resonances, since their contribution to the strength is small. If the strength is calculated only up to the energy at which resonances begin to be missed, the result is within the statistical errors quoted in the table above.

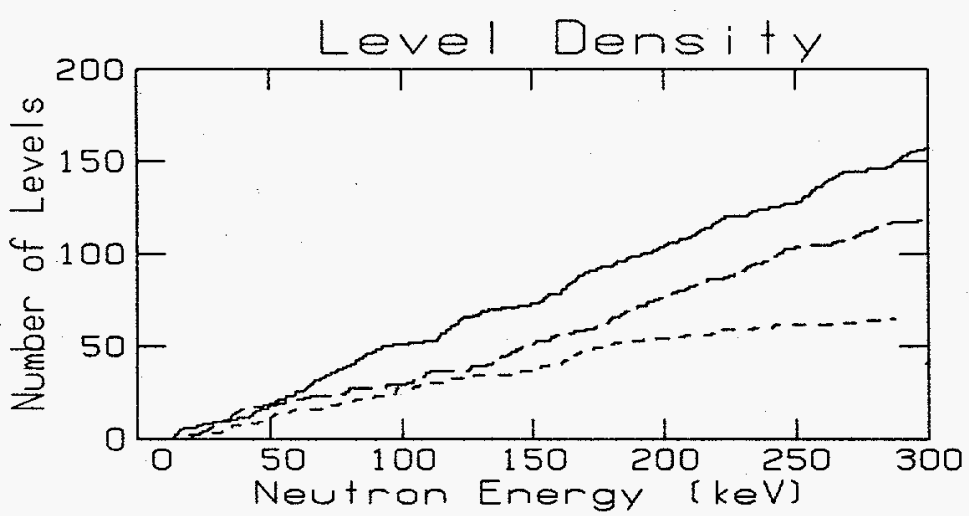

Figure 4. Cumulative number of levels versus neutron energy for the $s_{1 / 2}(-), p_{1 / 2}(--)$, and $p_{3 / 2}(-)$ resonances. The slope of the histograms gives the level density for that partial wave.

The distribution of levels between the two $p$ - wave spin states is inconsistent with the expected $2 J+1$ dependence. This may be, in part, a consequence of the criterion used to apportion resonances between the two states where, for some small resonances, the differences in $\chi^{2}$ values for different assumed $J$ - values were small and subject to random factors such as initial parameter estimates. Histograms similar to those in Fig. 4 for only levels of definite $J^{\pi}$ are consistent with the expected dependence, corroborating the suggestion of improper partitioning.

\section{External R-functions ( $R^{\text {ext }}$ )}

The $R^{\text {ext }}$ functions are related to the real part of the optical potential and their general energy trends provide a constraint on the optical model parameters. In this nuclide distinction can be made between $p_{1 / 2}$ and $p_{3 / 2}$ resonances for only $25 \%$ of the non $s$ - wave resonances, with consequent uncertainty in the strengths deduced therefrom. The $R^{\text {est }}$, however, can be deduced wherever a broad resonance of determined $J^{n}$ occurs and the number of such occurrences does not influence the uncertainty in the $R^{e x t}$ as much as in the case of the average resonance properties. The $R^{\text {ext }}$ parameters and uncertainties for both these partial waves have been determined at the energies of resonances of known spin and parity. Their energy dependencies are determined additionally by their influence on the background cross section throughout the energy range. At low energies the contribution to the off-resonance cross section is almost entirely due to $s$-wave potential scattering. As a consequence, the $s$-wave $R^{\text {ext }}$ parameters are well determined through their influence upon the potential scattering radius, $R_{\mathrm{o}}^{\prime}$ (See Eqn. (5)). A $10 \%$ change in the $s$-wave $\mathrm{R}^{\mathrm{ext}}$, for example, produces a visually distinguishable change in the total cross section in the $14-15 \mathrm{keV}$ energy region. The maximum uncertainty for this parameter for $s$ - waves, for the entire energy region, is $25 \%$. Since concentrations of the isotopic impurities of the sample are lower by one to two orders of magnitude, compared to ${ }^{122} S n$, their $s$ - wave contributions to the offresonance cross section will be correspondingly lower. Their contribution, through the $R^{e x t}$, has nonetheless been included by setting impurity $s$-wave $R^{\text {ext }}$ parameters equal to those found for ${ }^{122} S n$.

Changes in the $p$ - wave $R^{\text {ext }}$ parameters of a factor of two have no noticeable effect on the observed off-resonance cross section or resonance asymmetries in the low energy region. At higher energies the $p$-wave influences become very discernible with regard to both the off-resonance cross section and the interference asymmetry of large $p$-wave resonances. Since the $R_{\ell J}^{\text {ext }}$ are related to the real part of the optical model potential, their values and uncertainties will influence the required well depth. We established the uncertainties by manual variation of the parameter $\alpha$ until a visual comparison showed noticeable disagreement with those asymmetries and non-resonant cross sections obtained from least squares parameters, in a region where the largest resonance(s) of a given $J^{\pi}$ occurred. Thus, while at $50 \mathrm{keV}$ the $p$-wave parameters can be changed by a factor of 2 without noticeable change in the resonance asymmetry, at 80 $\mathrm{keV}$ the visually ascertained uncertainty is $50 \%$ and at 275 $\mathrm{keV}$ the uncertainty is approximately $10 \%$. This variation has been parametrized and is reflected in error bars shown in Fig 5. For clarity $p$ - wave error bars are shown for only the $p_{1 / 2}$, those for the $p_{3 / 2}$ being the same. Error bars for the $s$-waves are smaller than the symbol size. The $R^{\text {ext }}$ parameters for the contributing partial waves are given in Table IV where the uncertainties in $\alpha$ and $\beta$ are reflected through the value of the smooth $R$ - function, $\widetilde{R}_{\ell J}$, at the midpoint of the energy region. The upper and lower limits for the $R$-matrix strength, $\widetilde{s}_{\ell J}$, have been determined as discussed in Section V. A.

Table IV. Parametrization ${ }^{\dagger \dagger}$ for the external $R$ - functions:

\begin{tabular}{ccccc}
$J^{\pi}$ & $\alpha$ & $\beta(1 / \mathrm{MeV})$ & $\tilde{s}_{U}\left(\times 10^{+2}\right)$ & $\widetilde{R}(\bar{E})$ \\
\hline$s_{1 / 2}$ & 0.13 & 0.024 & $0.39(7)^{\dagger}$ & $0.13(1)$ \\
$p_{1 / 2}$ & -0.33 & 0.58 & $7.8(10)_{4.4}^{13}$ & $-0.24(10)$ \\
$p_{3 / 2}$ & -0.27 & 0.30 & $5.7(7)_{3.3}^{7.5}$ & $-0.22(10)$
\end{tabular}




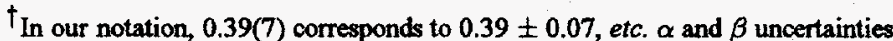
are discussed in the text. Upper and lower limits are established as in Table III.

"ISee equs. 4 and 7 for relationship of parameters to the R-function.

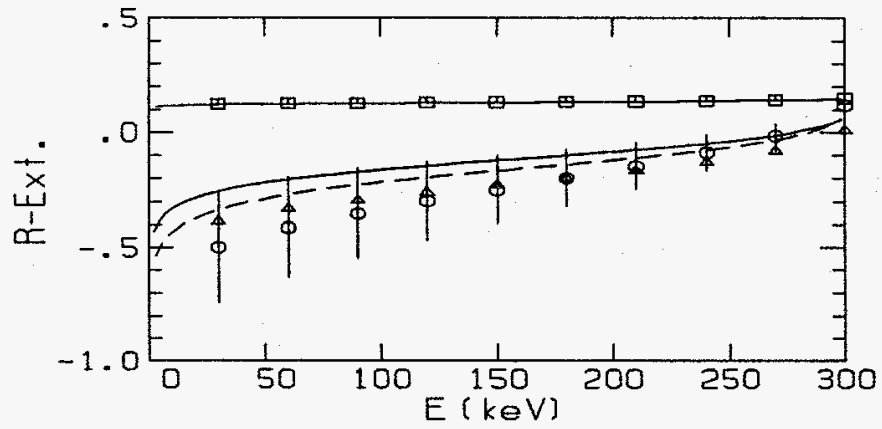

Figure 5. The External $R$-functions for the $s_{1 / 2}(\square), p_{1 / 2}(\odot)$, and $p_{3 / 2}(\Delta)$ partial waves. The error bars for the $p_{3 / 2}$ are comparable to those shown for the $p_{1 / 2}$. Smooth curves represent optical model predictions (dashed curve for $p_{1 / 2}$ ).

\section{VL. Average Parameters and the OMP}

Low energy neutron elastic scattering in the ${ }^{122} S n+n$ system serves as a probe of the optical model potential for each partial wave and is accomplished via the $R_{\ell J}^{\text {ext }}$ and $\widetilde{s}_{\ell J}$. The $R^{\text {ext }}$ provide the dominant contribution to the real part, $V_{r}$, of the optical potential and the strength function is related to the surface imaginary depth, $W_{D}$.

Energy-averaged scattering functions can be related to the average resonance parameters through the expression,

$$
<S_{\ell J}(E)>e^{2 i \phi_{\ell}(E)} \frac{\left[1+i P_{\ell}(E) R_{\ell J}(E)\right]}{\left[1-i P_{\ell}(E) R_{\ell J}(E)\right]}
$$

where the complex $R$ - function can be approximated ${ }^{7}$ by

$$
\boldsymbol{R}_{\ell J}(E)=\widetilde{R}_{\ell J}(E)+i \pi \widetilde{s}_{\ell J}(E) .
$$

The justification for the approximation and the details of the comparison of the averaged empirical functions with predicted scattering functions, $S_{\ell J}^{O M P}(E)$, are treated in more detail in Ref. 6.

Our procedure was to use a Woods-Saxon well with real, surface imaginary, and spin-orbit terms. We performed a least squares adjustment of the real and imaginary depths until the integrated strengths and $R_{\ell J}^{\text {ext }}$ predicted by the model agreed with our experimental values. The geometry of the model was taken from work on ${ }^{116} \mathrm{Sn}$ and ${ }^{120} \mathrm{Sn}$ by Guss et. al ${ }^{3}$. and held constant at the values shown in Table V. The well depth for the spin-orbit potential was likewise held constant in the fitting process.
Table V. Radius and Diffuseness parameters ${ }^{\dagger}$ for OMP.

\begin{tabular}{c|ccc}
\hline & $V_{r}$ & $W_{D}$ & $S O$ \\
\hline$r$ & 1.23 & 1.25 & 1.12 \\
$a$ & 0.66 & 0.54 & 0.50 \\
\hline
\end{tabular}

${ }^{\dagger}$ Values are all in $\mathrm{fm}$.

The predicted results for the $s$ - wave $R^{\text {ext }}$, shown by the solid curves in Fig. 5, are indistinguishable from the empirical values. In the case of $p$-waves the predicted results are systematically high throughout most of the region, but are within the error bars. (The dashed curve is to be compared with the $\odot$ values and the solid curve with the $\triangle$ values.) The latter have error bars of the same magnitude as those for the $p_{1 / 2}$. The agreement is good and the energy trends of the model predictions closely parallel the experimental trends.

The corresponding comparison for the $s_{1 / 2}$ integrated strength is presented in Fig. 6. The model representation in this case is good up to an energy of $240 \mathrm{keV}$.

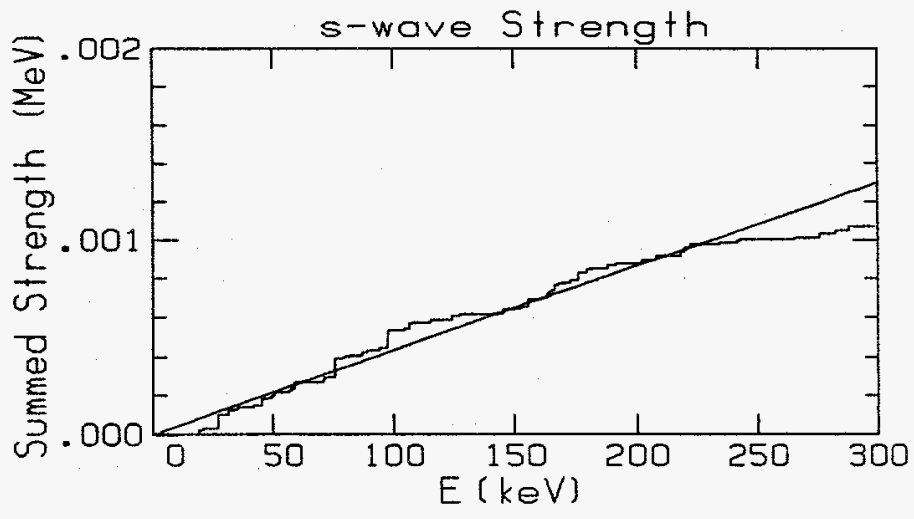

Figure 6. Experimental integrated strength and model predictions, based upon geometric and potential parameters in Tables V and VI.

The experimental results for the component $p$-wave integrated strengths have been presented earlier in the discussion of strength functions. The solid lines in Fig. 3 represent model predictions based upon parameters deduced from the least squares adjustment, applied separately for the two components. These are seen to describe the data well over the entire energy range. And, this description was achieved with virtually identical model parameters. However, the fits obtained for the average parameters for the two angular momentum states required different depths for both the real and surface imaginary potentials.

The average of the real well depths for $s$ - and $p$-waves is consistent with the parametrization found for similar isotopes of tin in the study of Guss, $e t . a l^{3}$, after suitable adjustments for isotope and energy differences. These results are presented in Table VI with the values from the model deduced by Guss, et. al. It should be noted that their model overestimated the 
$p$-wave strength functions by more than $40 \%$. When we used the geometry and well depths of Guss et. al. our model $R^{e x t}$ was too high for $s$-waves and too low for the $p$-waves, both by factors of appproximately two. An identical comparison held for the model $s$ - and $p$-wave strengths.

Table VI. Spherical Optical Model Parameters ${ }^{\dagger}$

\begin{tabular}{c|ccccc}
\multicolumn{4}{c}{ Present Work } & \multicolumn{2}{c}{ Guss, et. al. } \\
\multicolumn{1}{c}{$V_{r}$} & $W_{D}$ & $W_{S O}$ & $V_{r}$ & $W_{D}$ \\
\hline$s_{1 / 2}$ & 46.0 & 1.0 & 6.5 & 48.2 & 1.0 \\
$p_{1 / 2}$ & 50.0 & 6.2 & 6.5 & 48.2 & 1.0 \\
$p_{3 / 2}$ & 50.0 & 6.6 & 6.5 & 48.2 & 1.0 \\
\hline tWell depths are in MeV.
\end{tabular}

Most investigations aimed at elucidating the form of the optical model potential do not include low-energy information in the analysis. It should not be expected that these results would call for an $\ell$-dependence to the real well depth. In the present study all the data is low energy and one parameter, the $s$-wave smoothed $R$-function, is most precisely determined at energies approaching zero. We have in addition, information on the energy dependence of this parameter for both $\ell$ - waves (See Fig.5). Here, as in other similar studies ${ }^{4}$, we thus conclude that the real well depth must be deeper for $\mathrm{p}$-waves than for $\mathrm{s}$-waves to describe the low-energy neutron interaction.

\section{Discussion}

Because of the large number of stable isotopes of tin, this element has been studied extensively to gain information on the neutron-excess and energy dependencies of the parameters of the optical model potential (OMP). Differential elastic scattering and total cross sections for many of the isotopes of tin have been measured by Guss ${ }^{3}$, Rapaport ${ }^{8}$, and Harper ${ }^{9}$ and used to deduce these dependencies for the well depths of the potential. In another study which more closely approximates the present work, in type and energy range $(0.3-5.0 \mathrm{MeV})$, Harper et. al. ${ }^{10}$ deduced parameters which represent their total cross section measurements rather well in all but the $2-5 \mathrm{MeV}$ energy region. While there are small nuances of difference in analysis approach and the parameters obtained in the various studies, all have focused on a larger energy range and thus a more complete data set than in the present study. Conventional differential elastic scattering and total cross section measurements provide strong constraints on the optical model potential, but generally have little $\ell$ specificity. Because of the extent of the data in most studies of this type, the geometric parameters are well determined. Since their analyses consider data that is non-partial wave specific, it is expected that there may be parts of our average quantities which will not be reproduced by the complete parameter set from such studies.

In order to assure that the different geometries of the other studies could not account for the $\ell$-dependence we observed, we also searched for well depths corresponding to several of these geometries which would best represent all our data. The $\ell$-dependence persisted in each case, with only the magnitudes of the well depths changing with geometry. Thus it is seen in Table VII that in every case the difference between required $s$ - and $p$ - wave well depths is approximately $2-4 \mathrm{MeV}$ for the real potential and the imaginary potentials differ by more than $4 \mathrm{MeV}$. It should be noted that not all features of all data sets of the other studies have been properly described by their deduced parameters. The cross sections are low in some cases and high for others. This could be a manifestation of the need for an $\ell$-dependence, which our data clearly dictate.

Table VII. Optical Model Parameters for various geometries

\begin{tabular}{cccccccccc}
\hline & \multicolumn{3}{c}{ Guss $^{a}$} & \multicolumn{3}{c}{ Harper $^{b}$} & \multicolumn{3}{c}{ Rapaport $^{c}$} \\
& $s_{1 / 2}$ & $p_{1 / 2}$ & $p_{3 / 2}$ & $s_{1 / 2}$ & $p_{1 / 2}$ & $p_{3 / 2}$ & $s_{1 / 2}$ & $p_{1 / 2}$ & $p_{3 / 2}$ \\
$V_{r}(\mathrm{MeV})$ & 46.0 & 49.8 & 49.4 & 45.2 & 48.2 & 48.2 & 46.4 & 49.0 & 51.4 \\
$W_{D}(\mathrm{MeV})$ & 1.0 & 5.0 & 5.0 & 1.9 & 6.3 & 6.9 & 0.9 & 11.0 & 6.2 \\
$V_{S O}(\mathrm{MeV})$ & 6.5 & 6.5 & 6.5 & 5.5 & 5.5 & 5.5 & 6.2 & 6.2 & 6.2 \\
$\alpha_{V}\left(\mathrm{Mev}^{-1}\right)$ & & 0.30 & & & 0.27 & & & 0.36 & \\
$\alpha_{D}\left(\mathrm{Mev}^{-1}\right)$ & 0.0 & & & 0.053 & & & 0.52 & \\
\hline
\end{tabular}

$a_{V}=1.23 ; r_{D}=1.25 ; r_{S O}=1.12 ; a_{V}=0.66 ; a_{D}=0.54 ; a_{s O}=0.50$, all in $f m$ ${ }^{b} r_{V}=1.26 ; r_{D}=1.26 ; r_{S O}=1.12 ; a_{V}=0.58 ; a_{D}=0.40 ; a_{S O}=0.50$, all in $f m$ $c_{r_{V}}=1.20 ; r_{D}=1.32 ; r_{s o}=1.01 ; a_{V}=0.70 ; a_{D}=0.62 ; a_{s o}=0.76$, all in $f m$ $\alpha V$ and $\alpha_{D}$ give the energy dependence of the corresponding well depths

Some studies have included $\ell$-specific properties as parameters in the model, such as potential scattering radii or strength functions, but none have obtained information on these quantities through direct identification or observation of individual contributions to partial wave samples. For example, Timokhov, et. al. ${ }^{11}$ have modeled neutron capture cross sections and transmissions, for all the stable tin isotopes over the energy range $20-1400 \mathrm{keV}$, in the framework of the Hauser-Feshbach-Moldauer formalism. They have deduced strength functions and potential scattering radii for $s-$ and $p$-wave interactions. In the present study we have obtained potential scattering radii, in addition to the average scattering functions for each $\mathrm{J}^{\pi}$, according to the relation ${ }^{12}$,

$$
\mathrm{R}^{\prime}=\mathrm{a}_{\mathrm{c}}[1-(2 \ell+1) \widetilde{\mathrm{R}}(\mathrm{E}=0)]
$$

Comparison of results from these considerations are presented in Table VIII. They also deduced an OMP and found that not all data can be represented with a single potential. They did not, however, report an $\ell$-dependence.

Table VIII. Potential Scattering Radii 


\begin{tabular}{llll}
\hline $\mathrm{J}^{\pi}$ & Present & Timokhov, et. al. & Popov \\
\hline $1 / 2^{+}$ & $6.3(2)$ & $6.1(2)$ & $6.0(2)$ \\
$1 / 2^{-}$ & $8.9(2)$ & & \\
$3 / 2^{-}$ & $8.8(2)$ & & \\
$\mathrm{p}$ & & $8.6(3)$ & $9.3(2)$ \\
\hline
\end{tabular}

An increasing body of evidence is accumulating that one must impose an $\ell$-dependence to an optical model description of results of the analysis of high resolution low energy elastic neutron scattering cross sections ${ }^{4}$.

\section{Conclusions}

The tin isotopes have been the focus of the prediction that possible doonway structure may exist in this mass region, based on evidence presented many years ago for the presence of enhanced M1 strength in these isotopes. Though one might expect similar structure for s-waves, our analysis of high resolution neutron total cross section data for ${ }^{122} S n$ would suggest little support for this expectation, as seen from the cumulative plots of s-wave reduced neutron widths presented in Fig. 6. In earlier calculations, in which doorway structure was postulated in order to account for the low s-wave strength, Shakin ${ }^{13}$ predicted the doorways to lie at much higher energies than covered in the present analysis. We are thus able to conclude from this study that any possible doorway structure in these isotopes of tin must lie above 310 $\mathrm{keV}$.

Several studies have suggested the need for an $\ell$-dependence for the real well depth of the OMP, when analyzing low energy total cross section data. We find further evidence in this isotope of tin. Mahaux has recently suggested ${ }^{14}$ an alternate interpretation for this result in which a surface potential is included, thereby obviating the need for an $\ell$-dependence, an interpretation which finds more theoretical support than the former. Application of these ideas to this and other spin-zero isotopes of tin would provide clarification concerning this feature of low energy partial wave analysis of neutron total cross section data.

\section{Code Development}

\section{GUI:}

Besides the work on the ${ }^{122} \mathrm{Sn}$ we have also spent significant effort on computer-code development. In recent years, the computations associated with this project have all been ported to the RISC architecture because of the superior speed and memory capabilities. Since the RISC computer uses an $\mathrm{X}$-windows interface, and since the minimization codes are graphics intensive, we have developed a graphical user interface (GUI) for interactive analysis of neutron transmission data in the R-matrix framework using the Xwindows programming constructs. A suitable commercial package could not be located which provided the flexibility and customization desired to most effectively enhance the ability of this project to efficiently process and analyze these data from ORELA. A physics student has been involved in the project, gaining valuable experience in programming and in the analysis of neutron cross section data. A template now exists that will permit the routine incorporation of other computer codes into the interface, making possible the routine analysis of neutron cross section data.

\section{DOM minimization:}

Another computer code was written to develop the computational capability to describe certain experimental nuclear structure information with a specialized dispersive optical model (DOM) of the nuclear potential and to automate the computations so that the full physical range of parameter space of the model is explored in achieving the best representation of the data. This procedure, while not in the purest sense true in the current formulation, is known by the term "least squares fitting".

Several program segments were developed, each to perform a local task, and all subroutines were bound together to form the program called sphmsmin. The code was inspired by Mahaux and Sartor's paper on ${ }^{40} \mathrm{Ca}$. The Hartree - Fock, the volume imaginary and the surface imaginary potentials can have different geometries, namely, $\mathrm{r}_{\mathrm{H}}, \mathrm{a}_{\mathrm{H}}, \mathrm{r}_{\mathrm{iv}}, \mathrm{a}_{\mathrm{iv}}, \mathrm{r}_{\mathrm{D}}, \mathrm{a}_{\mathrm{D}}$. The imaginary volume integrals have the Brown-Rho form and begin at a binding energy, $E_{0}$. The Fermi energy, $E_{F}$, and all well depths are read in, and variables for use in the minimization subroutine are assigned, together with flags which determine which of these variables are to be varied in the minimization. After minimization a final pass is made with the "best" parameters to obtain the model strengths and $R$ - functions.

The code was written to constrain the model parameters by fitting the cumulative strength $(s)$, the external $\mathrm{R}$ - function $(R)$, and total cross section $(\sigma)$ simultaneously using a linear combination of contributions from the experimental quantities, as $\chi^{2}=\chi_{s}^{2}+\chi_{R}^{2}+\chi_{\sigma}^{2}$. This code will be incorporated with the GUI above to facilitate user interaction with the minimization routine.

\section{References}

\footnotetext{
${ }^{1}$ T. Fuketa, and J.A. Harvey, Oak Ridge National Laboratory Report No. ORNL-3425 (1962), (unpublished),p. 36.

${ }^{2}$ J. A. Harvey, and T. Fuketa, in Proceedings of Conference on Study of Nuclear Structure with Neutrons, edited by $M$.
} 
Neve de Mervergnies, P. Van Assche, and J. Vervier, (North Holland, Amsterdam, 1966), p. 527.

${ }^{3}$ P. P. Guss, R. C. Byrd, C. R. Howell, R. S. Pedroni, G. Tungate, and R. L. Walter, Phys. Rev C 39, 405 (1989). ${ }^{4} \mathrm{C}$. H. Johnson, in AIP Conference Proceedings 124 on Neutron-Nucleus Collisions, edited by J. Rapaport, R. W. Finlay,S. M. Grimes, and F. S. Dietrich, (AIP, New York, 1985), p. 446.

${ }^{5}$ N. M. Larson, Oak Ridge National Laboratory Report No. ORNL/TM-9179/R2, Oak Ridge, Tennessee, 1989 (unpublished).

${ }^{6}$ C. H. Johnson, R. F. Carlton, and R. R. Winters, Phys. Rev. C 39, 415 (1989).

${ }^{7}$ C. H. Johnson, C. Mahaux, and R. R. Winters, Phys. Rev. C 32, 359 (1985).

${ }^{8}$ J. Rapaport, M. Mirzaa, H. Hadizadeh, D. E. Bainum, and R. W. Finlay, Nucl. Phys. A 341, 56 (1980).

${ }^{9} \mathrm{R}$. W. Harper, J. L. Weil, and J. D. Brandenberger, Phys. Rev. C 30, 1454 (1984).

${ }^{10}$ R. W. Harper, T. W. Godfrey, and J. L. Weil, Phys. Rev. C 26, 1432 (1982).

${ }^{1}$ V. M. Timokhov, M. V. Bokhovko, A. G. Isakov, L. E. Kazakov, V. N. Kononov, G. N. Manturov, E. D. Poletaev, and V. G. Pronyaev, Sov. J. Nucl. Phys. 50, 375 (1989).

${ }^{12}$ A. B. Popov, and G. S. Samosvat, Proc. Int. Conf., Santa $\mathrm{Fe}, 1985$, p. 621 .

${ }^{13}$ C. Shakin, Ann. Phys., 22, 373 (1963).

${ }^{14}$ C. Mahaux and H. Ngo, Nucl. Phys. A431, (1984). 